\title{
Long-Term Memory Updating: The Reset-of-Encoding Hypothesis in List-Method Directed Forgetting
}

\author{
Bernhard Pastötter ${ }^{*}$, Tobias Tempel${ }^{1}$ and Karl-Heinz T. Bäum/2 \\ ${ }^{1}$ Department of Psychology, University of Trier, Trier, Germany, ${ }^{2}$ Department of Psychology, University of Regensburg, \\ Regensburg, Germany
}

People's memory for new information can be enhanced by cuing them to forget older information, as is shown in list-method directed forgetting (LMDF). In this task, people are cued to forget a previously studied list of items (list 1) and to learn a new list of items (list 2) instead. Such cuing typically enhances memory for the list 2 items and reduces memory for the list 1 items, which reflects effective long-term memory updating. This review focuses on the reset-of-encoding (ROE) hypothesis as a theoretical explanation of the list 2 enhancement effect in LMDF. The ROE hypothesis is based on the finding that encoding efficacy typically decreases with number of encoded items and assumes that providing a forget cue after study of some items (e.g., list 1) resets the encoding

Edited by:

Christoph T. Weidemann, Swansea University, United Kingdom

Reviewed by:

Geoff Ward,

University of Essex, United Kingdom Monika Undorf,

University of Mannheim, Germany

*Correspondence: Bernhard Pastötter pastoetter@uni-trier.de

Specialty section: This article was submitted to Cognitive Science, a section of the journal

Frontiers in Psychology

Received: 07 September 2017 Accepted: 14 November 2017 Published: 27 November 2017

Citation:

Pastötter B, Tempel T and Bäuml K-HT (2017) Long-Term Memory Updating: The Reset-of-Encoding Hypothesis in List-Method Directed Forgetting.

Front. Psychol. 8:2076.

doi: 10.3389/fpsyg.2017.02076 process and makes encoding of subsequent items (e.g., early list 2 items) as effective as encoding of previously studied (e.g., early list 1) items. The review provides an overview of current evidence for the ROE hypothesis. The evidence arose from recent behavioral, neuroscientific, and modeling studies that examined LMDF on both an item and a list level basis. The findings support the view that ROE plays a critical role for the list 2 enhancement effect in LMDF. Alternative explanations of the effect and the generalizability of ROE to other experimental tasks are discussed.

Keywords: long-term memory, episodic memory, directed forgetting, encoding, interference

\section{INTRODUCTION}

Long-term memory updating plays a vital role in creating an adaptive human memory system. According to Bjork (1978), such updating is critical because "everyday functioning requires that we keep our (episodic) memories reasonably current. To the degree that we do not somehow set aside or eliminate (irrelevant) information no longer needed we become confused, error prone, and inefficient" (p. 236). Indeed, goal-directed remembering of current or relevant information may fail because irrelevant information is retrieved and thus interferes with the retrieval of the relevant information (Bäuml, 2008). Effective long-term memory updating should reduce interference from irrelevant information and thus promote goal-directed remembering of the current or relevant information.

In the laboratory, memory updating can be studied with the list-method directed forgetting (LMDF) paradigm (Bjork, 1970, 1972). In this paradigm, participants study two lists of items (e.g., words, sentences, or pictures) and, after study of list 1 (L1), receive a cue either to forget or to 
continue remembering this list (Figure 1A). After study of list 2 (L2) and a short retention interval, a memory test for both lists is conducted, in which all participants are asked to recall the items of the two lists, irrespective of original cuing. The typical finding is that forget-cued participants recall more L2 items and fewer L1 items than remember-cued participants. The two effects of the forget cue are referred to as L2 enhancement (L2E) and L1 forgetting (L1F) in the following (Figure 1B; MacLeod, 1998; Bäuml et al., 2010; Sahakyan et al., 2013). This review focuses on the cognitive mechanisms underlying L2E, that is, the beneficial effect of intentional memory updating in the LMDF paradigm.

\section{FROM SINGLE- TO DUAL-MECHANISM ACCOUNTS OF LMDF}

Single-mechanism accounts of LMDF assume that L2E and L1F are the two sides of the same coin and are mediated by the same cognitive mechanism. For instance, the retrieval-inhibition account assumes that participants engage in active inhibitory processes in response to the forget cue. The inhibition impairs access to L1 context and, due to the resulting decrease in L1 items' interference level, enhances memory for L2 (Geiselman et al., 1983). Alternatively, the context-change account assumes that forget-cued participants deliberately change mental context between study of the two lists. Such change may impair access to L1 context, reduce the list's interference level, and thus improve memory for L2 (Sahakyan and Kelley, 2002). Although the two single-mechanism accounts thus differ in detail with regard to the exact nature of the mediating mechanism, they both take a retrieval view on L2E, attributing the effect to interference reduction at test (for further single-mechanism accounts, see MacLeod, 1998).

Several findings are in line with single-mechanism accounts of LMDF and the retrieval view on L2E. Bäuml and Kliegl (2013), for instance, showed that the forget cue reduces response latency during L2 recall and makes it similar to latency when a single list has been studied only. Because reduced response latency is assumed to reflect a reduction in participants' memory search set size (Wixted and Rohrer, 1993), the finding indicates more focused memory search during L2 recall in forget-cued participants, as caused by reduced interference from L1 (see also Bjork and Bjork, 1996; Sahakyan and Kelley, 2002). In contrast, there are also findings that are inconsistent with single-mechanism accounts of LMDF, for instance, reporting dissociations between L2E and L1F. These dissociations indicate that L2E can occur without L1F (e.g., Pastötter et al., 2016; Tempel and Frings, 2016) and L1F can occur without L2E (e.g., Sahakyan and Delaney, 2003; Pastötter and Bäuml, 2010). To explain these findings, dual-mechanism accounts of LMDF have been suggested, according to which both retrieval and encoding processes can contribute to LMDF (Sahakyan and Delaney, 2003; Pastötter and Bäuml, 2010; Pastötter et al., 2012).

One hypothesis reflecting such view is reset-of-encoding (ROE; Pastötter and Bäuml, 2010; Pastötter et al., 2012). The ROE hypothesis is based on the view that encoding efficacy of studied items decreases with number of encoded items, and is thus better for early than for later studied items. Such decrease may arise due to increases in working memory load or reduced attention during encoding when more and more items are encoded (Sederberg et al., 2006; Pastötter et al., 2008). Within a study list, this view is reflected in the well-known primacy effect, which demonstrates better memory for early than for middle and late studied list items (Deese and Kaufman, 1957; Murdock, 1962). The view is also reflected in the generally better memory for a first compared to a second study list, when two item lists were encoded in succession. Here the effect is mainly caused by the presence of a primacy effect in the first list and the absence (or reduction) of the effect in the second (e.g., Geiselman et al., 1983; Pastötter and Bäuml, 2010). The critical assumption of the ROE hypothesis then is that providing a forget cue after study of L1 resets the encoding process and makes the encoding of the early L2 items as effective as the encoding of the early L1 items. Two immediate predictions arise from such a view, one on an item and the other on a list level basis: The one prediction is that, after a forget cue, not only L1 but also L2 should show a primacy effect, leading to better memory for early than later studied L2 items. The other prediction is that (neural and modeling) parameters relating to encoding efficiency should change from L1 to L2 encoding in the remember condition but not in the forget condition.

The ROE hypothesis has been incorporated in the dualmechanism account of LMDF of Pastötter et al. (2012). According to this account, two mechanisms contribute to LMDF: a retrieval mechanism, i.e., retrieval inhibition, supposed to contribute to both L2E and L1F; and an encoding mechanism, i.e., ROE, supposed to contribute to L2E (of early L2 items) only. In the next paragraphs, we will focus on L2E and review current evidence for the ROE hypothesis.

\section{EVIDENCE FOR ROE}

First evidence for the ROE hypothesis arose from behavioral studies that examined items' serial position curves in LMDF on an item level basis. In more recent work, additional evidence for the ROE hypothesis emerged from neurocognitive, model-based, and motor learning studies addressing LMDF on a list level basis. We will review both lines of evidence for ROE.

\section{Evidence from Serial Position Curves}

First evidence that the forget cue in LMDF may have a selective enhancement effect for early L2 items arose in the studies by Geiselman et al. (1983) and Sahakyan and Foster (2009), reporting improved recall of early relative to later L2 items. On the basis of these findings, Pastötter and Bäuml (2010) ran a series of new LMDF experiments, in which they manipulated number and presentation rate of $\mathrm{L} 2$ items. At test, participants were instructed to recall L1 items first and L2 items second. Analysis of items' serial position curves showed a large recall enhancement effect for the early L2 items, but no reliable enhancement effect for the middle and late L2 items (Figure 1C). Neither number nor presentation rate of L2 items influenced the selective enhancement effect for the early L 2 items. On the basis of these findings, the ROE hypothesis was suggested, attributing 


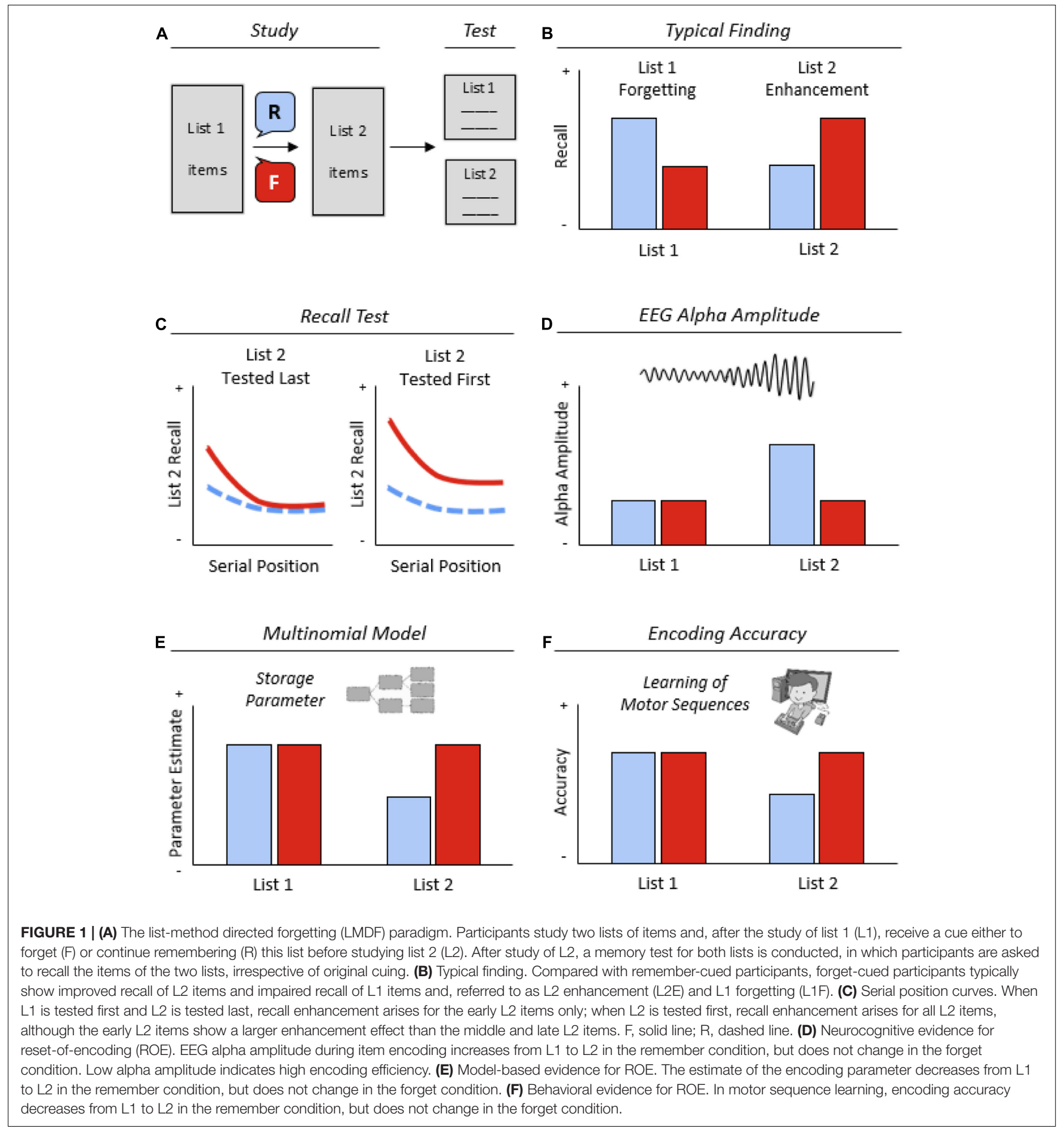

the selective enhancement effect to a reset of the encoding process in response to the forget cue. ${ }^{1}$

Further evidence for the ROE hypothesis arose from a study that examined the influence of the two lists' recall order at test

\footnotetext{
${ }^{1}$ Note that, regarding L1, no influence of items' serial learning position was observed. Indeed, all L1 items showed about the same amount of forgetting, which is consistent with the idea that L1F arises from reduced L1 context access (e.g., Sahakyan and Foster, 2009; Pastötter and Bäuml, 2010).
}

for LMDF effects (Pastötter et al., 2012). When L1 was recalled first and L2 was recalled second, a selective enhancement effect for the early L2 items arose, replicating previous findings by Pastötter and Bäuml (2010). In contrast, when L2 was recalled first, recall enhancement for all L2 items arose, although the early L2 items stilled showed a larger enhancement effect than did the middle and late L2 items (Figure 1C). These findings indicate that two factors can contribute to L2E: one factor that pertains 
to early L2 items only and is present regardless of list recall order, and a second factor that pertains to all L2 items and is present only if L2 is recalled first. The dual-mechanism account of Pastötter et al. (2012) suggests that the first factor is ROE and the second factor is interference reduction due to retrieval inhibition. In fact, interference reduction for (all) L2 items may be reduced when $\mathrm{L} 1$ is recalled first, because the preceding recall of L1 items can reactivate L1 study context and thus reinstate L1 items' interference potential (Bäuml and Samenieh, 2010, 2012).

Pastötter et al. (2016) reported further support for the ROE hypothesis by examining serial position effects when employing item recognition tests. In this study, a large enhancement effect in item recognition for the early L2 items was found, but no enhancement effects for the middle and late L2 items emerged, irrespective of list testing order. Because item recognition should be sensitive to ROE-induced improved encoding but be fairly insensitive to (a reduction in) proactive interference (see Kinnell and Dennis, 2011), the finding by Pastötter et al. (2016) corroborates the view that the selective enhancement effect for the early L2 items reflects ROE and is in line with Pastötter et al.'s (2012) dual-mechanism account.

\section{Neurocognitive Evidence}

Neurocognitive evidence for the ROE hypothesis arose from LMDF studies that examined EEG alpha oscillations during the encoding of the two item lists (Bäuml et al., 2008; Hanslmayr et al., 2012). In prior EEG work, brain oscillations at distinct frequencies were linked to memory function (Nyhus and Curran, 2010; Hanslmayr et al., 2016). In particular, in episodic memory, EEG alpha oscillations $(8-14 \mathrm{~Hz}$ ) were associated with encoding efficiency in both single- and multi-list studies, with increases of EEG alpha amplitude indicating impaired item encoding (Sederberg et al., 2006). Consistently, in several studies, alpha amplitude was found to increase with number of encoded items, which was attributed to increases in memory load and reduced attention during item encoding (Sederberg et al., 2006; Pastötter et al., 2008, 2011).

Examining the role of EEG alpha oscillations in LMDF, Hanslmayr et al. (2012) demonstrated that alpha amplitude during item encoding increases from L1 to L2 in the remember condition, but not in the forget condition (Figure 1D). In addition, Bäuml et al. (2008) showed that the difference in EEG alpha amplitude during L2 encoding between the forget and remember conditions is specifically related to $\mathrm{L} 2 \mathrm{E}$, but not to $\mathrm{L} 1 \mathrm{~F}$. Together, these neurocognitive findings indicate that the forget cue resets neural activity back to $\mathrm{L} 1$ level and thus improves the encoding (and remembering) of L2 items, which fits with the ROE hypothesis.

\section{Model-Based Evidence}

Model-based evidence for the ROE hypothesis arose from a study using multinomial modeling to investigate LMDF (Rummel et al., 2016). Multinomial models are a class of mathematical models that can be used to disentangle the cognitive processes underlying observable behavioral effects on the basis of categorical data (Batchelder and Riefer, 1999). Rummel et al. (2016) applied the storage-retrieval model (Rouder and Batchelder, 1998) to quantify the relative contribution of encoding and retrieval processes to LMDF. Two modifications of the standard paradigm were necessary to meet the model's assumptions. First, participants studied word pairs instead of single words and, second, the usual free recall test was followed by an additional cued recall test. In the free recall test, L1 was tested first and L2 was tested second.

Recall results demonstrated both reliable L2E and L1F in the free recall test. In the model-based analysis, both storage and retrieval parameters were estimated. Estimates of the storage parameter were specifically related to L2E, whereas estimates of the retrieval parameter were specifically related to L1F. The storage parameter decreased from L1 to L2 in the remember condition, but did not change in the forget condition (Figure 1E). The finding suggests impaired encoding from L1 to L2 in the remember condition but not in the forget condition, which is consistent with the ROE hypothesis. Moreover, the model-based dissociation between L2E and L1F supports dual-mechanism views on $\mathrm{LMDF}$ that attribute $\mathrm{LMDF}$ effects to different mechanisms.

\section{Evidence from Motor Sequence Learning}

Further direct evidence for the ROE hypothesis comes from a LMDF study by Tempel and Frings (2016) that investigated the effects of cuing on motor sequence learning on a list level basis. Participants learned two lists of sequential finger movements (SFMs). Each list consisted of five SFMs and each SFM consisted of four key presses on a computer keyboard, performed with three fingers of the right hand. Both accuracy in entering SFMs in the learning phase and correct recall of SFMs in the test phase were analyzed as a function of cuing condition, separately for the two lists. L2 was tested after L1. Three results emerged. First, encoding accuracy decreased from L1 to L2 in the remember condition, but did not change in the forget condition (Figure 1F). Second, forget-cued participants correctly recalled more L2 SFMs than remember-cued participants. Third, L2 encoding accuracy mediated L2 recall enhancement. The findings indicate that LMDF effects generalize from verbal learning to non-verbal motor learning. In particular, they support the ROE hypothesis, suggesting that the forget cue resets motor encoding quality for L2 SFMs back to L1 level.

\section{ALTERNATIVE ENCODING HYPOTHESES}

In addition to the ROE hypothesis, two other hypotheses exist in the LMDF literature that attribute L2E to beneficial encoding: the strategy-change hypothesis and the selectiverehearsal hypothesis. The strategy-change hypothesis assumes that L2E arises from a change in forget-cued participants' encoding strategy (Sahakyan and Delaney, 2003). The idea is that the forget cue induces evaluation of participants' L1 encoding strategy and a shift to a better L2 encoding strategy, leading to more elaborate encoding of L2 items in the forget than in the remember condition (Sahakyan et al., 2004). Because, arguably, different encoding strategies should affect the encoding for all L2 items (Glanzer and Koppenaal, 1977), the strategy-change 
hypothesis predicts non-selective enhancement for all L2 items, which is inconsistent with the finding of a selective enhancement effect for the early L2 items. To reconcile the hypothesis with this finding, the restriction would have to be made that forgetcued participants shift to an encoding strategy that is beneficial for early L2 items only, or shift to more elaborate encoding for early L2 items and then switch back to less elaborate encoding for middle and late L2 items, which of course contrasts with Glanzer and Koppenaal's (1977) finding.

The selective-rehearsal hypothesis assumes that during L2 encoding remember-cued participants rehearse both L1 and L2 items in working memory, whereas forget-cued participants rehearse L2 items only, which improves subsequent memory for L2 at the expense of L1 (Bjork, 1970). On the item level, the hypothesis assumes that the enhancement should be largest for early L2 items and the forgetting should be largest for late L1 items, claiming that, once the forget cue is provided, mainly the late L1 items are deleted from the rehearsal buffer and the rehearsal starts over again with the encoding of the early L2 items (Bruce and Papay, 1970). Although the selectiverehearsal hypothesis is consistent with the finding of a selective enhancement effect for the early L2 items, it is inconsistent with the findings that (i) L1F is typically absent in item recognition (e.g., Benjamin, 2006; Pastötter et al., 2016), and (ii) L1F is typically non-selective in recall tests (e.g., Sahakyan and Foster, 2009; Pastötter and Bäuml, 2010).

\section{FROM LMDF TO OTHER EXPERIMENTAL TASKS}

The ROE hypothesis may not be restricted to the LMDF paradigm but generalize to other experimental tasks. For instance, several findings suggest that ROE can play a role in experimental tasks involving retrieval practice. Retrieval practice can have a number of beneficial effects for memory and learning (see Roediger et al., 2011). One such effect, referred to as the forward effect of testing in the literature, is that retrieval practice of previously studied information can increase retention of subsequently studied new information (Szpunar et al., 2008; for a review, see Pastötter and Bäuml, 2014). Arguably, the forward effect may partly be mediated by some form of self-induced forget instruction,

\section{REFERENCES}

Abel, M., and Bäuml, K.-H. T. (2016). Retrieval practice can eliminate listmethod directed forgetting. Mem. Cogn. 44, 15-23. doi: 10.3758/s13421-0150539-x

Batchelder, W. H., and Riefer, D. M. (1999). Theoretical and empirical review of multinomial process tree modeling. Psychon. Bull. Rev. 6, 57-86. doi: 10.3758/ BF03210812

Bäuml, K.-H. (2008). "Inhibitory processes," in Cognitive Psychology of Memory, Learning and Memory: A Comprehensive Reference, Vol. 2, ed. H. L. Roediger III (Oxford: Elsevier), 195-220.

Bäuml, K.-H., Hanslmayr, S., Pastötter, B., and Klimesch, W. (2008). Oscillatory correlates of intentional updating in episodic memory. Neuroimage 41, 596-604. doi: 10.1016/j.neuroimage.2008.02.053 inducing participants to think that the practiced information is no longer needed and thus can be forgotten (Szpunar et al., 2007; see also Abel and Bäuml, 2016). If so, retrieval practice should show the same basic effects as a forget cue, including ROE. Results from EEG studies support the proposal showing that retrieval practice (of episodic, semantic, and autobiographical memories) between the study of lists can disrupt alpha amplitude increases from the encoding of earlier to later lists, which may reflect ROE on a list level basis (Pastötter et al., 2008, 2011). To examine the role of ROE for the forward effect of testing or other experimental tasks more thoroughly, future work is needed to investigate the presence of ROE on an item level basis.

\section{CONCLUSION}

Several lines of LMDF studies support the ROE hypothesis. First, on the list level, there is consistent evidence from neurocognitive, model-based, and motor-learning studies indicating that the forget cue can reset post-cue encoding processes, making the encoding of L2 items about as effective as the encoding of L1 items. Second, on the item level, there is conclusive evidence from analysis of serial position data showing that, in response to a forget cue, L2 items at early serial learning positions are subject to selective enhancement and thus show a primacy effect similar to early L1 items. All of these findings converge on the view that ROE plays a critical role in LMDF and long-term memory updating. Finally, there is evidence that ROE can also be involved in other experimental tasks, which points to a more general role of ROE in creating an adaptive memory system.

\section{AUTHOR CONTRIBUTIONS}

BP drafted the manuscript. TT and K-HB provided critical revisions.

\section{FUNDING}

The publication was funded by the Open Access Fund of Universität Trier and the German Research Foundation (DFG) within the Open Access Publishing funding program.

Bäuml, K.-H., Pastötter, B., and Hanslmayr, S. (2010). Binding and inhibition in episodic memory - cognitive, emotional, and neural processes. Neurosci. Biobehav. Rev. 34, 1047-1054. doi: 10.1016/j.neubiorev.2009.04.005

Bäuml, K.-H. T., and Kliegl, O. (2013). The critical role of retrieval processes in release from proactive interference. J. Mem. Lang. 68, 39-53. doi: 10.1016/j.jml. 2012.07.006

Bäuml, K.-H. T., and Samenieh, A. (2010). The two faces of memory retrieval. Psychol. Sci. 21, 793-795. doi: 10.1177/0956797610370162

Bäuml, K.-H. T., and Samenieh, A. (2012). Selective memory retrieval can impair and improve retrieval of other memories. J. Exp. Psychol. Learn. Mem. Cogn. 38, 488-494. doi: 10.1037/a0025683

Benjamin, A. S. (2006). The effects of list-method directed forgetting on recognition memory. Psychon. Bull. Rev. 13, 831-836. doi: 10.3758/BF0319 4005 
Bjork, E. L., and Bjork, R. A. (1996). Continuing influences of to-be-forgotten information. Conscious. Cogn. 5, 176-196. doi: 10.1006/ccog.1996.0011

Bjork, R. A. (1970). Positive forgetting: the noninterference of items intentionally forgotten. J. Verbal Learn. Verbal Behav. 9, 255-268. doi: 10.1016/S00225371(70)80059-7

Bjork, R. A. (1972). "Theoretical implications of directed forgetting," in Coding Processes in Human Memory, eds A. W. Melton and E. Martin (Washington, DC: Winston), 217-235.

Bjork, R. A. (1978). "The updating of human memory," in The Psychology of Learning and Motivation, Vol. 12, ed. G. H. Bower (New York, NY: Academic Press), 235-259.

Bruce, D., and Papay, J. P. (1970). Primacy effect in single-trial free recall. J. Verbal Learn. Verbal Behav. 9, 473-486. doi: 10.1016/S0022-5371(70)80090-1

Deese, J., and Kaufman, R. A. (1957). Serial effects in recall of unorganized and sequentially organized verbal material. J. Exp. Psychol. 54, 180-187. doi: $10.1037 / \mathrm{h} 0040536$

Geiselman, R. E., Bjork, R. A., and Fishman, D. (1983). Disrupted retrieval in directed forgetting: a link with posthypnotic amnesia. J. Exp. Psychol. Gen. 112, 58-72. doi: 10.1037/0096-3445.112.1.58

Glanzer, M., and Koppenaal, L. (1977). The effect of encoding tasks on free recall: stages and levels. J. Verbal Learn. Verbal Behav. 16, 21-28. doi: 10.1016/S00225371(77)80004-2

Hanslmayr, S., Staresina, B. P., and Bowman, H. (2016). Oscillations and episodic memory: addressing the synchronization/desynchronization conundrum. Trends Neurosci. 39, 16-25. doi: 10.1016/j.tins.2015.11.004

Hanslmayr, S., Volberg, G., Wimber, M., Oehler, N., Staudigl, T., Hartmann, T., et al. (2012). Prefrontally driven down-regulation of neural synchrony mediates goal-directed forgetting. J. Neurosci. 32, 14742-14751. doi: 10.1523/ JNEUROSCI.1777-12.2012

Kinnell, A., and Dennis, S. (2011). The list length effect in recognition memory: an analysis of potential confounds. Mem. Cogn. 39, 348-363. doi: 10.3758/s13421010-0007-6

MacLeod, C. M. (1998). "Directed forgetting," in Intentional Forgetting: Interdisciplinary Approaches, eds J. M. Golding and C. M. MacLeod (Mahwah, NJ: Erlbaum), 1-57.

Murdock, B. J. (1962). The serial position effect of free recall. J. Exp. Psychol. 64, 482-488. doi: 10.1037/h0045106

Nyhus, E., and Curran, T. (2010). Functional role of gamma and theta oscillations in episodic memory. Neurosci. Biobehav. Rev. 34, 1023-1035. doi: 10.1016/j. neubiorev.2009.12.014

Pastötter, B., and Bäuml, K.-H. (2010). Amount of postcue encoding predicts amount of directed forgetting. J. Exp. Psychol. Learn. Mem. Cogn. 36, 54-65. doi: 10.1037/a0017406

Pastötter, B., and Bäuml, K.-H. (2014). Retrieval practice enhances new learning: the forward effect of testing. Front. Psychol. 5:286. doi: 10.3389/fpsyg.2014. 00286

Pastötter, B., Bäuml, K.-H., and Hanslmayr, S. (2008). Oscillatory brain activity before and after an internal context change - evidence for a reset of encoding processes. Neuroimage 43, 173-181. doi: 10.1016/j.neuroimage.2008. 07.005

Pastötter, B., Kliegl, O., and Bäuml, K.-H. T. (2012). List-method directed forgetting: the forget cue improves both encoding and retrieval of postcue information. Mem. Cogn. 40, 861-873. doi: 10.3758/s13421-0120206-4

Pastötter, B., Kliegl, O., and Bäuml, K.-H. T. (2016). List-method directed forgetting: evidence for the reset-of-encoding hypothesis employing itemrecognition testing. Memory 24, 63-74. doi: 10.1080/09658211.2014.985589
Pastötter, B., Schicker, S., Niedernhuber, J., and Bäuml, K.-H. T. (2011). Retrieval during learning facilitates subsequent memory encoding. J. Exp. Psychol. Learn. Mem. Cogn. 37, 287-297. doi: 10.1037/a0021801

Roediger, H. L., Putnam, A. L., and Smith, M. A. (2011). "Ten benefits of testing and their applications to educational practice," in Psychology of Learning and Motivation: Cognition in Education, eds J. Mestre and B. Ross (Oxford: Elsevier), $1-36$.

Rouder, J. N., and Batchelder, W. H. (1998). "Multinomial models for measuring storage and retrieval processes in paired associate learning," in Recent Progress in Mathematical Psychology: Psychophysics, Knowledge, Representation, Cognition, and Measurement, eds C. E. Dowling, F. S. Roberts, and P. Theuns (New York, NY: Psychology Press), 195-225.

Rummel, J., Marevic, I., and Kuhlmann, B. G. (2016). Investigating storage and retrieval processes of directed forgetting: a model-based approach. J. Exp. Psychol. Learn. Mem. Cogn. 42, 1526-1543. doi: 10.1037/xlm0000266

Sahakyan, L., and Delaney, P. F. (2003). Can encoding differences explain the benefits of directed forgetting in the list method paradigm? J. Mem. Lang. 48, 195-206. doi: 10.1016/S0749-596X(02)00524-7

Sahakyan, L., Delaney, P. F., Foster, N. L., and Abushanab, B. (2013). "Listmethod directed forgetting in cognitive and clinical research: a theoretical and methodological review," in The Psychology of Learning and Motivation, ed. B. Ross, Vol. 59, (Philadelphia, PA: Elsevier), 131-189. doi: 10.1016/B978-0-12407187-2.00004-6

Sahakyan, L., Delaney, P. F., and Kelley, C. M. (2004). Self-evaluation as a moderating factor in strategy change in directed forgetting benefits. Psychon. Bull. Rev. 11, 131-136. doi: 10.3758/BF03206472

Sahakyan, L., and Foster, N. L. (2009). Intentional forgetting of actions: comparison of list-method and item-method directed forgetting. J. Mem. Lang. 61, 134-152. doi: 10.1016/j.jml.2009.02.006

Sahakyan, L., and Kelley, C. M. (2002). A contextual change account of the directed forgetting effect. J. Exp. Psychol. Learn. Mem. Cogn. 28, 1064-1072. doi: 10.1037/ 0278-7393.28.6.1064

Sederberg, P. B., Gauthier, L. V., Terushkin, V., Miller, J. F., Barnathan, J. A., and Kahana, M. J. (2006). Oscillatory correlates of the primacy effect in episodic memory. Neuroimage 32, 1422-1431. doi: 10.1016/j.neuroimage.2006.04.223

Szpunar, K. K., McDermott, K. B., and Roediger, H. L. (2007). Expectation of a final cumulative test enhances long-term retention. Mem. Cogn. 35, 1007-1013. doi: 10.3758/BF03193473

Szpunar, K. K., McDermott, K. B., and Roediger, H. L. (2008). Testing during study insulates against the buildup of proactive interference. J. Exp. Psychol. Learn. Mem. Cogn. 34, 1392-1399. doi: 10.1037/a0013082

Tempel, T., and Frings, C. (2016). Directed forgetting benefits motor sequence encoding. Mem. Cogn. 44, 413-419. doi: 10.3758/s13421-015-0565-8

Wixted, J. T., and Rohrer, D. (1993). Proactive interference and the dynamics of free recall. J. Exp. Psychol. Learn. Mem. Cogn. 19, 1024-1039. doi: 10.1037/02787393.19.5.1024

Conflict of Interest Statement: The authors declare that the research was conducted in the absence of any commercial or financial relationships that could be construed as a potential conflict of interest.

Copyright (c) 2017 Pastötter, Tempel and Bäuml. This is an open-access article distributed under the terms of the Creative Commons Attribution License (CC BY). The use, distribution or reproduction in other forums is permitted, provided the original author(s) or licensor are credited and that the original publication in this journal is cited, in accordance with accepted academic practice. No use, distribution or reproduction is permitted which does not comply with these terms. 